http://dx.doi.org/10.35381/r.k.v6i1.1442

\title{
Sistema de rehabilitación de motricidad post intervención por síndrome del túnel carpiano
}

\section{Define a post-intervention motor rehabilitation system for carpal tunnel syndrome}

Elvis Francisco Sinchiguano-Sánchez

francosanto 90@hotmail.com

Investigador Independiente, Chimborazo

Ecuador

https://orcid.org/0000-0002-9397-4247

Andrea Estefania Cuñez-Yánez

andrea-cy2@hotmail.com

Investigador independiente, Chimborazo

Ecuador

https://orcid.org/0000-0003-0418-6124

Eduardo Francisco García-Cabezas

egarcia@espoch.edu.ec

Escuela Superior Politécnica de Chimborazo. Grupo de Investigación \& vinculación

"AUTOPRO", Chimborazo

Ecuador

https://orcid.org/0000-0002-3547-472X

Juan Carlos Cayán-Martínez

jcayan@espoch.edu.ec

Escuela Superior Politécnica de Chimborazo. Grupo de Investigación \& vinculación

"AUTOPRO", Chimborazo

Ecuador

https://orcid.org/0000-0001-9573-3706

Recepción: 30 de julio 2021

Revisado: 20 de agosto 2021

Aprobación: 15 de octubre 2021

Publicación: 01 de noviembre 2021 


$$
\begin{gathered}
\text { Revista Arbitrada Interdisciplinaria KOINONIA } \\
\text { Año VI. Vol VI. Nº1. Edición Especial: Administración. } 2021 \\
\text { Hecho el depósito de Ley: FA2016000010 } \\
\text { ISSN: 2542-3088 } \\
\text { FUNDACIÓN KOINONIA (F.K). Santa Ana de Coro. Venezuela. }
\end{gathered}
$$

Elvis Francisco Sinchiguano-Sánchez; Andrea Estefania Cuñez-Yánez; Eduardo Francisco García-Cabezas;

Juan Carlos Cayán-Martínez

\title{
RESUMEN
}

La definición de la propuesta tecnológica se efectuó con un análisis documentalbibliográfico, con un enfoque de tipo cuantitativo, es cuasi experimental. Se planteó como objetivo general definir un sistema de rehabilitación de motricidad post intervención por síndrome del túnel carpiano. Además, se apoyó con la metodología Stage Gate, la cual va de la mano con la gestión de innovación diseñada, que permite desarrollar diferentes actividades de un proceso las cuales son cinco puertas de validación y cinco etapas de avance, al aplicar la metodología un equipo multifuncional aprueba el paso por cada Etapa - Puerta, incrementando la tasa de éxito e implementado la toma de decisiones. Se concluye, que el protocolo de rehabilitación es sustancial realizar un estudio bibliográfico para tener más información, ya sea en el ámbito tecnológico, médico, terapéutico, con esto se permitirá tener una selección de ejercicios óptimos y para generar la elaboración de un sistema de rehabilitación.

Descriptores: Terapia ocupacional; ergonomía; rehabilitación médica. (Palabras tomadas del Tesauro UNESCO).

\begin{abstract}
The definition of the technological proposal was made with a documentary-bibliographic analysis, with a quantitative approach, it is quasi-experimental. The general objective was to define a post-intervention motor rehabilitation system for carpal tunnel syndrome. In addition, it was supported by the Stage Gate methodology, which goes hand in hand with the designed innovation management, which allows the development of different activities of a process which are five validation doors and five stages of progress, when a team applies the methodology Multifunctional approves the passage through each Stage Door, increasing the success rate and implementing decision-making. It is concluded that the rehabilitation protocol is substantial to carry out a bibliographic study to have more information, whether in the technological, medical, therapeutic fields, with this it will be possible to have a selection of optimal exercises and to generate the elaboration of a rehabilitation system.
\end{abstract}

Descriptors: Occupational therapy; ergonomics; medical rehabilitation. (Words taken from the UNESCO Thesaurus). 


$$
\begin{gathered}
\text { Revista Arbitrada Interdisciplinaria KOINONIA } \\
\text { Año VI. Vol VI. Nº1. Edición Especial: Administración. } 2021 \\
\text { Hecho el depósito de Ley: FA2016000010 } \\
\text { ISSN: 2542-3088 } \\
\text { FUNDACIÓN KOINONIA (F.K). Santa Ana de Coro. Venezuela. }
\end{gathered}
$$

Elvis Francisco Sinchiguano-Sánchez; Andrea Estefania Cuñez-Yánez; Eduardo Francisco García-Cabezas;

Juan Carlos Cayán-Martínez

\section{INTRODUCCIÓN}

En la actualidad la revolución tecnológica durante las últimas décadas ha avanzado de una manera acelerada permitiendo desarrollar nuevas oportunidades y dando soluciones acertadas. Existen una gran variedad de softwares que contribuyen en la automatización de las maquinarias en la industria, en procesos productivos, rehabilitación, generando información inmediata mejorando así el control de la producción, seguridad y calidad en sus productos. A causa del desarrollo tecnológico los campos de aplicación se han incrementado en la industria alimenticia, farmacéutica, química, aeronáutico, entre otros. Los resultados obtenidos en cada una de estas áreas demuestran lo indispensable que se vuelve en la actualidad puesto que permite resolver problemas que anteriormente eran imposibles.

En este sentido, uno de ello es el síndrome de túnel carpiano según estudios muestra que afecta a un sin número de personas que trabajan ya sea en oficina, fábricas y en construcciones debido a que sufren lesiones muy comunes en las manos. Esta neuropatía provoca una discapacidad sensitiva y motora con repercusión en la esfera biológica, psicológica, social y económica, con una larga y complicada recuperación postquirúrgica (Cabrera González y Roche González, 2021, p.369). EI STC se da porque se realiza movimientos repetitivos, en muchas ocasiones se puede corregir sin intervención quirúrgica pero cuando no se toma las medidas preventivas es necesario intervención quirúrgica. Post intervención del STC se requiere seguir un protocolo de ejercicios para la rehabilitación por lo tanto se ha estudiado cuales son los más esenciales como es: flexión, extensión, desviación radial, desviación cubital, puño y semi puño. De acuerdo a Jiménez del Barrio, et al., (2016):

El síndrome del túnel carpiano (STC) se define como el conjunto de signos y síntomas que se producen por la lesión del nervio mediano a nivel de la muñeca. El STC consiste en la compresión del nervio mediano a su paso por el túnel carpiano. (p.591). 
Entre las diferentes actividades laborables que han sido establecidas en la mayoría como generadoras del Síndrome de túnel carpiano, se han encontrado: los músicos que realizan movimientos repetitivos e incomodos con las manos, deportistas en el área del tenis que realizan movimientos cortos, profesionales de la salud, profesionales especializados que utilizan ordenadores con teclado y mouse en donde ejecutan actividades con un reducido número de músculos, y causadas como consecuencia de otras patologías.

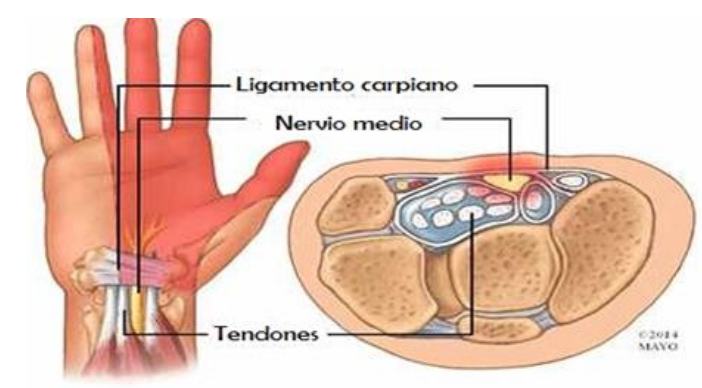

Figura 1. Anatomía Síndrome del túnel carpiano Fuente: https://images.app.goo.gl/q1jVx51dL5wrw7hw6

El síndrome de túnel carpiano nace de la presión excesiva en el nervio mediano del canal carpiano, afectando en estructuras anatómicas como ligamentos, tendones y músculos, lo cual repercute en el funcionamiento correcto del nervio mediano de la mano. Las personas que padecen de esta patología presentan dolores o malestares leves como: parestesias $u$ hormigueo de la mano, centrada en los dedos primarios de la mano y en la palma de la mano dominante del individuo, debilidad prensil del dedo pulgar e índice debido al atrofia de los nervios. La mano es un órgano fundamental en la historia evolutiva del hombre desde el enfoque social como biológico, de igual forma facilita en distintas actividades que están combinadas como es construir, crear, dibujar, etc. El Síndrome del Túnel Carpiano se lo identifica como una neuropatía periférica que perturba 
la correcta operatividad de la mano. EI STC fue por primera vez citado en 1833, por Ormerand el cual se lo asocio con un dolor nocturno y parestesias, sin embargo, fue en 1930, cuando se efectuó la primera operación exitosa por Learmont. (Rodríguez Menéndez et al., 2020, p.70).

Al transcurrir el tiempo sufren una decadencia laboral causado por el síndrome. El STC se encuentra diagnosticado en tres etapas: leve donde se corrige mediante ejercicios la postura y descanso, moderado se requiere un tratamiento con un profesional de la salud y grave donde se encuentran los síntomas prominentes y la única solución es la intervención quirúrgica. En el año 2016 se registraron 611 enfermedades profesionales al Seguro de Riesgo de Trabajo y de estos, 177 que equivale al 28,94\% son de riesgo tipo ergonómico y 56 casos que equivale al 9,18\% son por síndrome de túnel carpiano. (Ayala, 2018, p.17; citado en IESS, 2016).

Por otro lado, en el mercado existen diferentes softwares que facilitan el diseño de sistemas, permitiendo tener una perspectiva real de cómo sería el prototipo con características específicas como la selección del material, análisis estático, factor de seguridad mediante el software SolidWorks. En lo que es la programación y la transferencia de contenido como son instrucciones se hace uso del software Arduino IDE. El Arduino IDE (Entorno de desarrollo integrado) es una plataforma electrónica de código abierto, está establecida en software y hardware libre esto quiere decir que son accesible para todo el público que desee modificar.Tiene un lenguaje escrito de programación Java, el programa radica en editor de código, compilar depurador, interfaz gráfica. En el IDE permite escribir el código para programar lo que es la tarjeta, de igual forma el IDE valida el código para proceder a compilar y cargar al microcontrolador. (Amaya, 2020, p.22). Con el software LabVIEW permite dar un entorno gráfico con adquisición y presentación de datos. Dicho software permite controlar el sistema completo a través de navegadores de Internet mediante WEB Server. Database, Datasocket y WEB Server, son componentes que brinda ésta plataforma de programación. (Fernández Padrón, et al.,2012, p.52) 


$$
\begin{gathered}
\text { Revista Arbitrada Interdisciplinaria KOINONIA } \\
\text { Año VI. Vol VI. Nº1. Edición Especial: Administración. } 2021 \\
\text { Hecho el depósito de Ley: FA2016000010 } \\
\text { ISSN: 2542-3088 } \\
\text { FUNDACIÓN KOINONIA (F.K). Santa Ana de Coro. Venezuela. }
\end{gathered}
$$

Elvis Francisco Sinchiguano-Sánchez; Andrea Estefania Cuñez-Yánez; Eduardo Francisco García-Cabezas;

La definición de un sistema electromecánico está pensada con el fin de ayudar en la rehabilitación post intervención del STC donde considera varios factores como es la selección de materiales, selección de actuadores, selección de sistemas de transmisión, selección de microcontrolador que sean amigables con la persona que lo use. El sistema electromecánico genera un impacto positivo en la sociedad que permite tener accesibilidad a un programa de rehabilitación con una eficiente disponibilidad post intervención, ayudando de esta manera a recuperar la motricidad de mano y dedos en tiempo reducido, de igual forma facilitando así su incorporación inmediata a sus actividades laborales y sociales. Las terapias basadas en las tareas asistivas, tienen el efecto de reducir los errores de movimiento, ayudando al usuario a finalizar el ejercicio. (Mancisidor, et al., 2018, p.184)

Es sustancial resaltar la aportación tecnológica de última generación en el ámbito de diseño y producción de equipos de rehabilitación, debido a que facilitan a los profesionales de la salud en su desenvolvimiento laboral y de igual forma a los pacientes que requieren de esta asistencia en su rehabilitación. Al respecto, Peña Ayala, et al, (2018) plantea:

Uno de los campos de aplicación de la ergonomía es la biomecánica, ciencia que estudia el cuerpo humano, sus movimientos y limitaciones, y analiza las destrezas motoras. Esta área ayuda a la interpretación correcta del cuerpo humano para tener un mayor provecho del diseño de producto o estancia de trabajo y así evitar lesiones. (p66)

Por todo lo señalado se propone como objetivo general definir un sistema de rehabilitación de motricidad post intervención por síndrome del túnel carpiano. 


\section{METODOLOGÍA}

La definición de la propuesta tecnológica se efectuó con un análisis bibliográfico anteriormente detallado, la investigación tiene un enfoque del tipo cuantitativo, es cuasi experimental, el cual se asigna a un cierto número de sujetos que forman parte de la investigación de manera aleatoria, el diseño se debe realizar mediante un software que permitirá tener una idea previa a lo que será la implementación, de igual forma el diseño es de tipo observacional debido a que permite analizar las variables más adelante.

La metodología Stage Gate, va de la mano con la gestión de innovación diseñada, la cual permite desarrollar diferentes actividades de un proceso las cuales son cinco puertas de validación y cinco etapas de avance, al aplicar la metodología un equipo multifuncional aprueba el paso por cada Etapa - Puerta, incrementando la tasa de éxito e implementado la toma de decisiones. Detalla que un proceso de innovación "estándar y básico" tiene al menos cinco fases que son interactivas y simultáneas: generación de ideas, concepto del producto, desarrollo del producto, implementación del producto.(Cooper, Edgett y Kleinschmidt,2002). 
Revista Arbitrada Interdisciplinaria KOINONIA

Año VI. Vol VI. Nº1. Edición Especial: Administración. 2021

Hecho el depósito de Ley: FA2016000010

ISSN: 2542-3088

FUNDACIÓN KOINONIA (F.K). Santa Ana de Coro. Venezuela.

Elvis Francisco Sinchiguano-Sánchez; Andrea Estefania Cuñez-Yánez; Eduardo Francisco García-Cabezas;

Juan Carlos Cayán-Martínez

\section{Metodología Gates}

Fase de descubrimiento

La idea parte de la necesidad de obtener un sistema electromecánico post intervención del túnel carpiano donde se tenga un protocolo de rehabilitación adecuado para los diferentes pacientes.
Fase de alcance

La idea parte en la propuesta tecnológica que se ajuste a los diferentes requerimientos, de acuerdo a los datos arrojados posteriormente se puede comercializar el producto.

\section{Fase de desarrollo}

En esta fase se realiza el sistema electromecánico con ayuda de diferentes softwares ya sea en el diseño como en la programación.
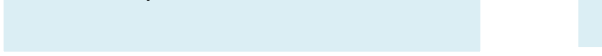

de

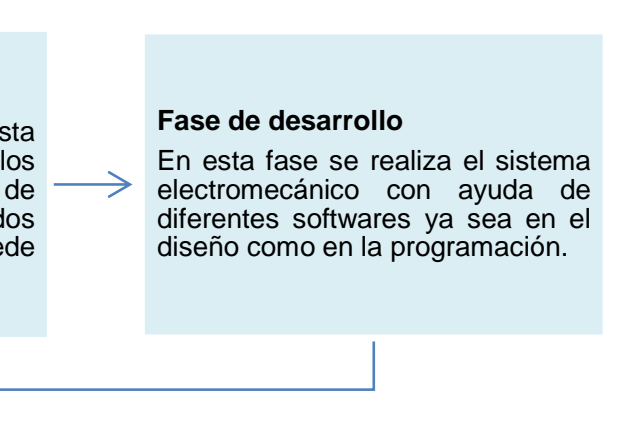

\[ \text { Fase de pruebas y validación } \]
La validación de la investigación
se la da de acuerdo a los datos
arrojados en los diferentes
softwares y alcance del sistema
electromecánico para la
rehabilitación.

\section{Fase de caso de negocio}

En esta fase se la puede desarrollar una vez implementado y se pueda conocer en el ámbito de costos y todos lo utilizado para el desarrollo del sistema.

\section{Fase de lanzamiento}

Posteriormente implementación a la considerar el lanzamiento del producto a mayor escala ya sea nacional como internacional.

\section{Gráfico 1.}

Elaboración: Los autores. 


$$
\begin{gathered}
\text { Revista Arbitrada Interdisciplinaria KOINONIA } \\
\text { Año VI. Vol VI. Nº1. Edición Especial: Administración. } 2021 \\
\text { Hecho el depósito de Ley: FA2016000010 } \\
\text { ISSN: 2542-3088 } \\
\text { FUNDACIÓN KOINONIA (F.K). Santa Ana de Coro. Venezuela. }
\end{gathered}
$$

Elvis Francisco Sinchiguano-Sánchez; Andrea Estefania Cuñez-Yánez; Eduardo Francisco García-Cabezas;

\section{RESULTADOS}

Por medio de la respectiva revisión e investigación documental y bibliográfica, se da lugar al desarrollo del diseño mecánico del sistema, con sus respectivas características, buscando que cumplan con los requisitos esperados. En este apartado se indicará el diseño mecánico generado por medio de la estructuración realizada a partir del siguiente gráfico.

\section{Propuesta teórica del diseño}

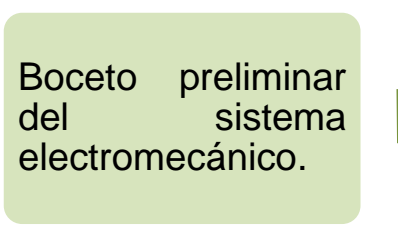

\section{Programación de entornos virtuales del procedimiento de rehabilitación} seleccionado
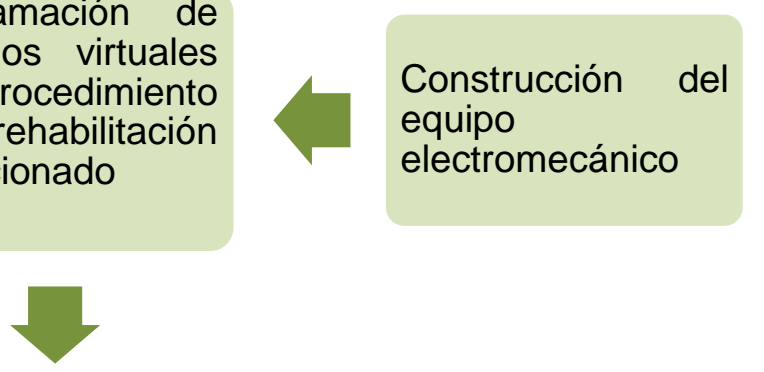

$\begin{array}{lr}\text { Comprobación } & \text { y } \\ \text { corrección } & \text { de } \\ \text { funcionalidad } & \text { del } \\ \text { diseño físico. } & \end{array}$

Vinculación del equipo electromecánico y programación. diseño

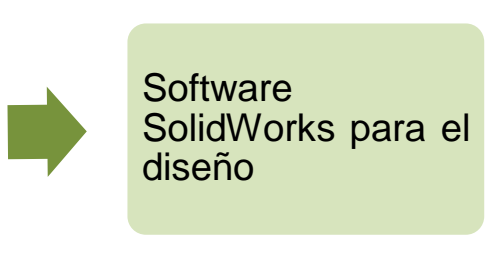

$\begin{array}{lr}\text { Simulación } & \text { y } \\ \text { verificación } & \text { de } \\ \text { funcionalidad } & \text { del } \\ \text { diseño } & \text { en } \\ \text { SolidWorks }\end{array}$

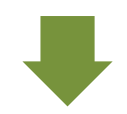
Análisis materiales y costos para la elaboración del sistema electromecánico.

\section{Gráfica 2.}

Elaboración: Los autores. 


$$
\begin{gathered}
\text { Revista Arbitrada Interdisciplinaria KOINONIA } \\
\text { Año VI. Vol VI. Nº1. Edición Especial: Administración. } 2021 \\
\text { Hecho el depósito de Ley: FA2016000010 } \\
\text { ISSN: 2542-3088 } \\
\text { FUNDACIÓN KOINONIA (F.K). Santa Ana de Coro. Venezuela. }
\end{gathered}
$$

Elvis Francisco Sinchiguano-Sánchez; Andrea Estefania Cuñez-Yánez; Eduardo Francisco García-Cabezas;

Diseño conceptual, el dispositivo debe cumplir con el concepto establecido para un sistema de rehabilitación que son ya dados por los principios de funcionabilidad, los mismos que debe tener los siguientes aspectos: Ergonomía, funcionabilidad, transportabilidad, cumplimiento del protocolo establecido. Los dedos cuentan con tres falanges (distal, media y proximal), sin embargo, el dedo pulgar tiene falange proximal y distal. En lo que respecta los cuatros dedos de la mano a excepción del dedo pulgar tienen un movimiento de flexión en la extensión normal de los dedos claramente lo realizan de manera conjunta y recta tocando la palma. El movimiento abducción los dedos se aíslan con un ángulo de $20^{\circ}$ y en aducción se juntan. (Velázquez, 2007) Selección del tipo de movimiento, todos los movimientos de la mano post intervención del STC parten de movimientos generales simples, estos movimientos se recuperan por medio de la rehabilitación después de haberlos perdido en la intervención por STC, se ha estudiado los procesos de rehabilitación medica donde se extrajo las secuencias que contengan los movimientos generales más acertados, buscando que los movimientos no requieran asistencia médica diaria. Los movimientos generales de la muñeca seleccionados son: flexión, extensión, desviación radial y desviación cubital. El movimiento de los dedos en agarre y semi agarre grupal, por otro lado, una secuencia dedo por dedo. 


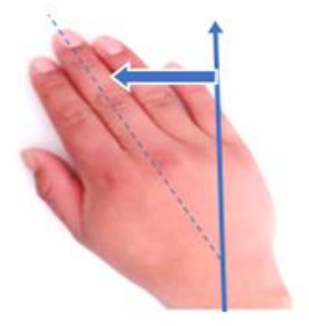

DESVIACIÓN RADLAL

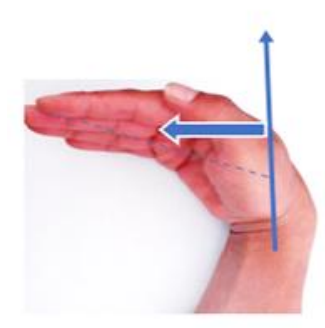

FLEXIÓN

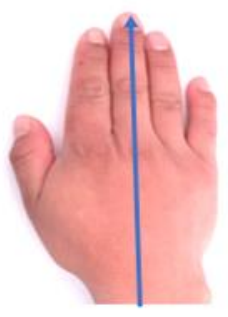

NORMAL

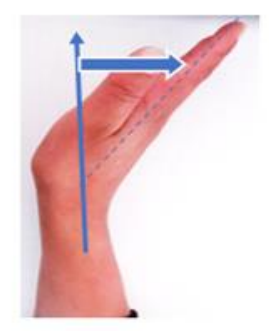

EXTENCIÓN

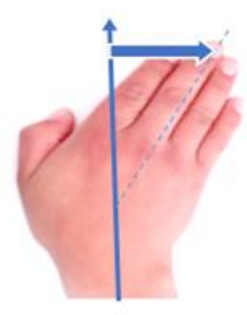

DESVLACIÓN CUNITAL

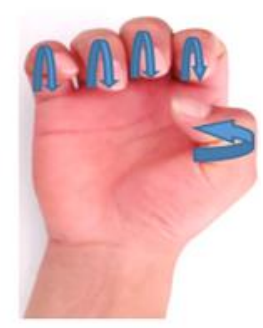

SEMI AGARRE

Figura 2. Movimientos generales

Elaboración: Los autores.

Adquisición de medidas antropométricas de la mano, la biometría conforma diferentes rasgos fisiológicos que diferencian a un ser humano de otro en este caso todo lo que engloba a la geometría de la mano ya sea en medidas y características. Conocer un promedio en medidas geométricas de la mano es sustancial debido a que nos permitirá establecer ciertos parámetros previo al diseño. Para el diseño del sistema electromecánico se realizó una base de datos donde se obtenga las medidas de las manos y dedos, con esto se logrará establecer un rango de movimientos para la funcionalidad del sistema. 
Protocolo y recolección de datos, la recolección de los datos de las medidas de las manos se efectuó en 23 personas donde se dio recomendaciones de cómo se debe realizar y llenar la base de datos con esto se obtendrá orden y a su vez permitirá obtener un máximo y mínimo con respecto a las medidas adquiridas.

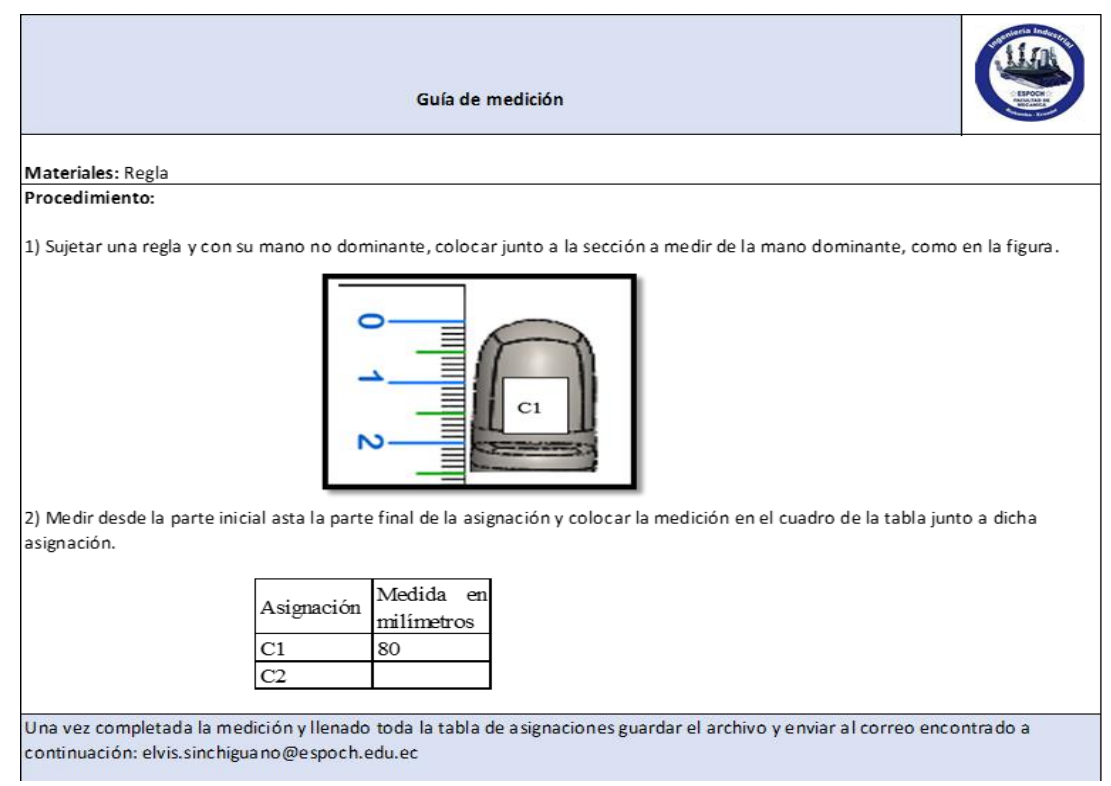

Figura 3. Guía de recolección de medidas Elaboración: Los autores.

A través de la recolección de datos se obtiene las medidas necesarias para el diseño mecánico del sistema electromecánico lo que permite trabajar con medidas reales para lo que será su uso. Esta se aplicó a un grupo de 23 estudiantes de la Escuela Superior Politécnica de Chimborazo de la carrera de Ingeniería Industrial. 
Revista Arbitrada Interdisciplinaria KOINONIA

Año VI. Vol VI. N 1 . Edición Especial: Administración. 2021

Hecho el depósito de Ley: FA2016000010

ISSN: 2542-3088

FUNDACIÓN KOINONIA (F.K). Santa Ana de Coro. Venezuela.

Elvis Francisco Sinchiguano-Sánchez; Andrea Estefania Cuñez-Yánez; Eduardo Francisco García-Cabezas;

Juan Carlos Cayán-Martínez

Dimensionamiento de la Mano

Seleccione con una $\mathrm{X}$
\begin{tabular}{|l|l|}
\hline \multicolumn{2}{|c|}{ Género } \\
\hline Masculino & \\
\hline Femenino & \\
\hline
\end{tabular}

Llenar los datos

Edad

Dedos

\begin{tabular}{|l|l|l|l|l|l|l|l|l|l|}
\hline \multicolumn{2}{|c|}{ Pulgar (mm) } & \multicolumn{2}{|c|}{ Índice (mm) } & \multicolumn{2}{|c|}{ Medio (mm) } & \multicolumn{2}{|c|}{ Anular (mm) } & \multicolumn{2}{|l|}{ Mefique (mm) } \\
\hline A1 & & B1 & & C1 & & D1 & & E1 & \\
\hline A2 & & B2 & & C2 & & D2 & & E2 & \\
\hline & & B3 & & C3 & & D3 & & E3 & \\
\hline
\end{tabular}
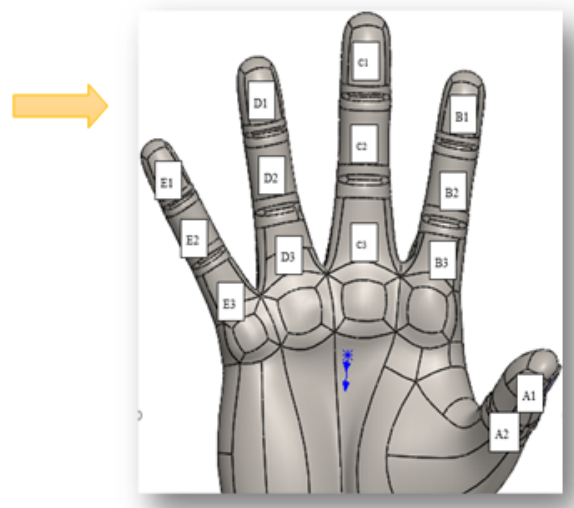

Mano, palma

\begin{tabular}{|l|l|l|}
\hline \multicolumn{1}{|c|}{ Detalle } & \multicolumn{2}{c|}{ Medida (mm) } \\
\hline Longitud de mano & F & \\
\hline Longitud palma de mano & G & \\
\hline Anchura de mano & H & \\
\hline Anchura palma de mano & I & \\
\hline Diámetro empuñera & J & \\
\hline
\end{tabular}
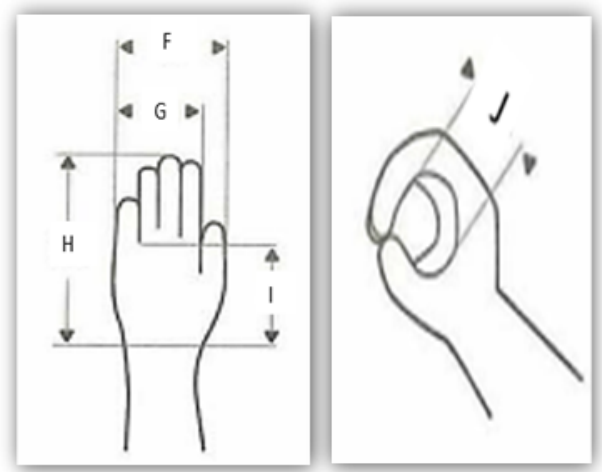

Figura 4. Base de datos para obtención de medidas. Elaboración: Los autores. 
Una vez indicado todo el proceso se procede a obtener las medidas de los dedos tanto el máximo como el mínimo, que posteriormente se utilizó para el diseño del prototipo considerando siempre que todas las personas tenemos diferentes medidas propias lo que nos hace diferentes unos de otros.

\section{Tabla 1.}

Rango de longitudes de los dedos.

\begin{tabular}{|c|c|c|}
\hline \multicolumn{3}{|c|}{ LONGITUD DE DEDOS } \\
\hline Dedo & Longitud $\mathbf{m i n}$. (mm) & Longitud máx. (mm) \\
\hline Pulgar & 69 & 42 \\
\hline Índice & 94 & 55 \\
\hline Medio & 109 & 61 \\
\hline Anular & 104 & 54 \\
\hline Meñique & 89 & 38 \\
\hline
\end{tabular}

Elaboración: Los autores.

Ángulos de movimiento de las manos, Conocer los ángulos de movimiento de la mano y dedos fue sustancial para la creación del diseño porque nos permite tener una idea general y establecer un protocolo de ángulos que una persona puede realizar. La mano desarrolla ángulos flexión- extensión de abducción-aducción en todas las articulaciones para aquello se tomó datos bibliográficos ya establecidos. 


\section{Tabla 2.}

Ángulos promedio de movilidad.

\begin{tabular}{|l|c|}
\hline \multicolumn{2}{|c|}{ PROMEDIOS DE MOVILIDAD DE LOS ÁNGULOS DE LA MANO } \\
\hline \multicolumn{1}{|c|}{ MOVIMIENTO } & PROMEDIO GRADOS ( $\left.{ }^{\circ}\right)$ \\
\hline Flexión & 70 \\
\hline Extensión & 55 \\
\hline Desviación radial & 42 \\
\hline Desviación cubital & 42 \\
\hline
\end{tabular}

Elaboración: Los autores.

Selección sistema de transmisión, para la realización de los movimientos requeridos de la mano se ha seleccionado el sistema de tornillos sin fin, en la sección A se implementa dos tornillos sin fin para la realización del movimiento horizontal y el movimiento vertical como se indica. En la sección A, se establece un sistema de transmisión de eje sin fin solo para transporte lineal como se indica en el diagrama siguiente, el punto $E$ es trasladado en forma vertical por medio del sistema $C D$ mientras que es el sistema $A B$ traslada en sentido horizontal todo el sistema $C D$ y el punto $E$ en conjunto, esto permite colocar la mano en los ángulos requeridos para cada uno de los movimientos establecidos en el protocolo de rehabilitación.

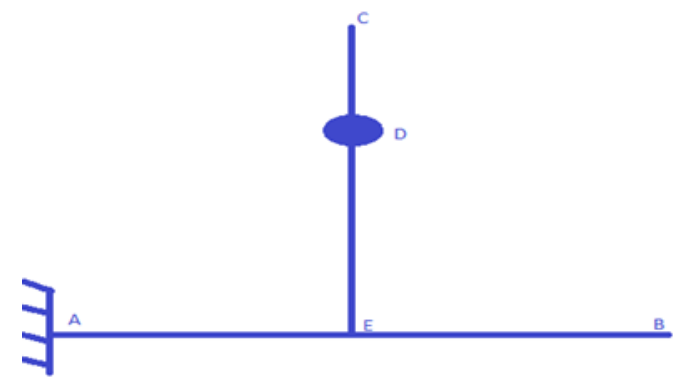

Figura 5. Mecanismo de transmisión de la sección A. Elaboración: Los autores. 
Revista Arbitrada Interdisciplinaria KOINONIA

Año VI. Vol VI. N 1 . Edición Especial: Administración. 2021

Hecho el depósito de Ley: FA2016000010

ISSN: 2542-3088

FUNDACIÓN KOINONIA (F.K). Santa Ana de Coro. Venezuela.

Elvis Francisco Sinchiguano-Sánchez; Andrea Estefania Cuñez-Yánez; Eduardo Francisco García-Cabezas;

Juan Carlos Cayán-Martínez

Para la sección B establece el sistema indicado a continuación, en este mecanismo se realiza el traslado del punto $\mathrm{C}$ a lo largo del sistema $\mathrm{AB}$ permitiendo el movimiento de los dedos en la sección correspondiente, esto permite realizar los movimientos dirigidos para la rehabilitación.

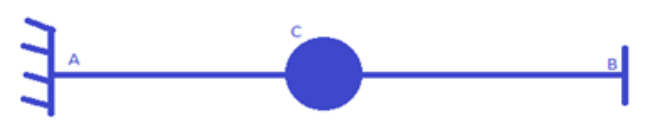

Figura 6. Mecanismo de transmisión de la sección B Elaboración: Los autores.

Selección del tornillo Sin Fin, se seleccionó dos tipos de tornillo sin fin debido a sus características individuales tanto físicas como individuales:

Tabla 3.

Características del tornillo sin fin.

\begin{tabular}{|c|c|c|c|}
\hline Características & Unidad & Tipo 1 & Tipo 2 \\
\hline Material & - & Acero inoxidable & $\begin{array}{c}\text { Acero } \\
\text { inoxidable }\end{array}$ \\
\hline Hilos & - & 4 & 1 \\
\hline $\begin{array}{c}\text { Avance por } \\
\text { revolución }\end{array}$ & milímetro & 8 & 2 \\
\hline Costo & Dólares/300mm & 15,99 & 15,99 \\
\hline $\begin{array}{c}\text { Capacidad } \\
\text { adquisitiva }\end{array}$ & - & Medio alta & Alta \\
\hline $\begin{array}{c}\text { Dimensiones } \\
\text { (diámetro) }\end{array}$ & milímetro & 8 & 8 \\
\hline Paso por vuelta & paso & 200 & 200 \\
\hline Ángulo por paso & grado & 1,8 & 1,8 \\
\hline
\end{tabular}

Elaboración: Los autores. 
El tornillo de cuatro hilos tiene un avance rápido de ocho milímetros por revolución permitiéndole recorrer mayor distancia en menor tiempo con respecto al de un hilo, pero al recorrer una mayor distancia disminuye su grado de posicionamiento, debido a esto se tiene el tornillo sin fin de cuatro hilos en la estación A ya que el grado de exigencia en precisión no es alta y se puede variar en cierta medida el desplazamiento. En la estación B se tiene el tornillo sin fin de un hilo permitiendo tener un mayor grado de control tanto en avance y tiempos. La característica de uno y cuatro hilos es la cantidad de canales que tiene el eje en la cual se impulsará la superficie roscada de la tuerca para su avance.

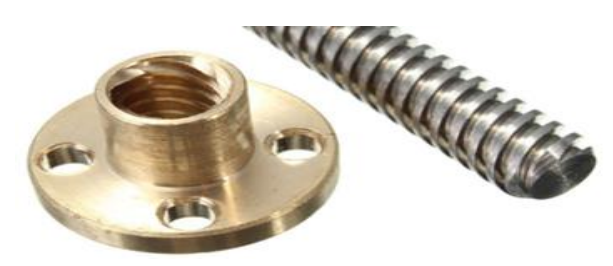

Figura 7. Tornillo sin fin de cuatro hilos Elaboración: Los autores.

Los autores. Selección de actuadores, para este sistema se seleccionó los motores paso a paso NEMA 17 debido a las diferentes ventajas que aporta para el desarrollo del sistema electromecánico como son: La precisión, donde el tipo de motores se puede controlar su avance por medio de los pasos que da por vuelta permitiendo ser preciso en la cantidad recorrida en cada movimiento evitando la variabilidad excesiva de los otros tipos de motores. Capacidad, por medio de las características de construcción puede ejercer la fuerza necesaria para los trabajos requeridos para este tipo de motor. Dimensiones, cuenta con unas dimensiones adaptables para cualquier instancia lo que amplía las áreas de su aplicación y otras características más como su precio, existencia en el mercado, eje de metal, puertos y drivers para su control y otros. En la siguiente 
tabla se muestra las características técnicas tomadas en cuenta para su selección como actuador para el sistema electromecánico.

Tabla .4

Características técnicas generales del motor paso a paso NEMA 17 POLOLU

\begin{tabular}{|l|l|l|}
\hline \multicolumn{1}{|c|}{ CARACTERÍSTICAS } & \multicolumn{1}{c|}{ UNIDAD } & \multicolumn{1}{c|}{ VALOR } \\
\hline Voltaje de Trabajo & Voltios & 4 \\
\hline Tipo de motor & Paso a paso bipolar & - \\
\hline Longitud del eje & milímetro & 25 \\
\hline Diámetro del Eje & milímetro & 5 \\
\hline Material del Eje & metal & \\
\hline Torque motor & Kg/cm & 3,2 \\
\hline Potencia & amperio & 1,2 \\
\hline Dimensiones & milímetro & $42^{*} 42^{*} 38$ \\
\hline Peso & gramo & 350 \\
\hline Paso por vuelta & paso & 200 \\
\hline Ángulo por paso & grado & 1,8 \\
\hline
\end{tabular}

Por medio de 7 motores NEMA 17 se debería, realizar los movimientos de todo el sistema electromecánico, dos destinados para los movimientos generales de la muñeca y cinco para los movimientos de los dedos un motor por cada dedo. 
Revista Arbitrada Interdisciplinaria KOINONIA

Año VI. Vol VI. N${ }^{\circ}$. Edición Especial: Administración. 2021

Hecho el depósito de Ley: FA2016000010

ISSN: 2542-3088

FUNDACIÓN KOINONIA (F.K). Santa Ana de Coro. Venezuela.

Elvis Francisco Sinchiguano-Sánchez; Andrea Estefania Cuñez-Yánez; Eduardo Francisco García-Cabezas;

Juan Carlos Cayán-Martínez

Selección del controlador, se seleccionó el Arduino UNO por motivo que tiene un fácil ingreso de código para el manejo de los actuadores, de adquisición inmediata, debido a la cantidad de actuadores requeridos resulto excelente para dicho control.

\section{Tabla 5.}

Características del Arduino UNO.

\begin{tabular}{|c|c|c|}
\hline Arduino UNO & Descripción & Especificaciones \\
\hline & $\begin{array}{l}\text { Tiene } 12 \text { pines digitales, } 6 \\
\text { analógicas, resonador } \\
\text { cerámico, conexión USB, } \\
\text { alimentador eléctrico y un } \\
\text { botón de reinicio, con solo } \\
\text { la conexión a PC el } \\
\text { programa previo instalado } \\
\text { se pone en función. }\end{array}$ & $\begin{array}{l}\text { Compatible con la aplicación de } \\
\text { Arduino } \\
14 \text { entradas y salidas Digitales } \\
6 \text { entradas analógicas } \\
6 \text { canales de PWM } \\
\text { Frecuencia de reloj de } 12 \text { Mhz. } \\
2 \text { alimentaciones de } 5 \text { Volts } \\
1 \text { entrada Vin, soporta de 9V- } \\
12 \mathrm{~V}\end{array}$ \\
\hline
\end{tabular}

Elaboración: Los autores. 
Diseño CAD, el diseño del sistema electromecánico inició con el estudio bibliográfico de diferentes estructuras ya existentes en el mercado, el estudio de las férulas que recomiendan los doctores a los pacientes post intervención del STC, los movimientos que se sugiere efectuar para comenzar a recupera movilidad, así mismo se revisó videos donde se muestra de manera más didacta una serie de ejercicio post intervención del STC, entonces se procedió a la selección y planteamiento de los movimientos requeridos para ejecutar el protocolo de rehabilitación con los datos obtenidos anteriormente se podrá tener límites máximos y mínimos de los movimientos, el diseño también se desarrolló con el principio de las máquinas CNC, debido a que su mecanismo permite obtener los movimientos requeridos, una de sus características es que tienes diferentes direcciones programables ya sea en línea recta o con una trayectoria circular.

Al sistema electromecánico se lo divide en dos secciones: La sección A encargada de generar los movimientos generales de la muñeca y la sección B dedicada a los movimientos generales de los dedos. Para ello se diseñó un sistema electromecánico que cumpla con los requerimientos establecidos, principalmente el funcionamiento de los motores paso a paso con el tornillo sin fin.

Estación A del sistema electromecánico, está constituida por sistemas de movimientos lineales que realizan el proceso de rehabilitación de la muñeca, para generar estos movimientos los tornillos están acoplado al eje del motor paso a paso, de tal forma que al momento de girar juntamente con tornillo sin fin se genera el desplazamiento lineal del acople tuerca de cobre del tornillo. 


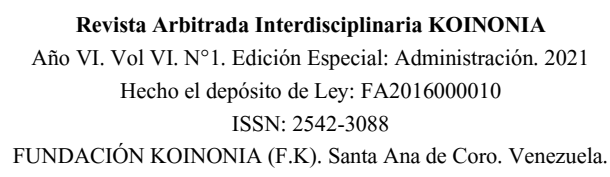

Elvis Francisco Sinchiguano-Sánchez; Andrea Estefania Cuñez-Yánez; Eduardo Francisco García-Cabezas; Juan Carlos Cayán-Martínez

Sección 1, está constituido por el movimiento en el plano horizontal en el Eje X, para permitir en la muñeca de la mano tener una rehabilitación en cuanto al movimiento radial y cubital. Dentro de su diseño para el deslizamiento se tiene ejes rotativos como son los tornillos sin fin que este sujeto a los acoples lineales conectándose directamente del otro extremo con el eje del motor paso a paso, admitiendo trasladar un punto de izquierda a derecha o de derecha a izquierda, las longitudes se encuentran orientadas para cumplir con las características de funcionalidad establecidas.

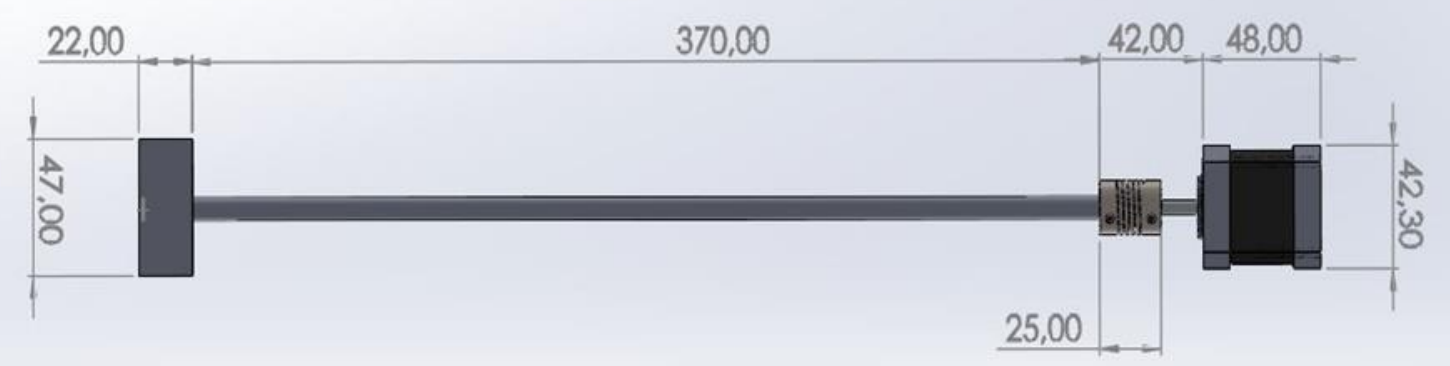

Figura 8. Diseño Sección 1.

Elaboración: Los autores. 
Sección 2, en esta sección el diseño esta dado para tener movimiento lineal en el plano vertical al eje $\mathrm{Y}$, el cual tiene para su deslizamiento 3 ejes que ayudara a tener estabilidad, así mismo cuenta con un tornillo sin fin y acoples lineales, también se diseñó una estructura que evite la fricción con la sección 1, de igual forma se consideró ya la estructura en donde se sujetara la muñeca para realizar el movimiento tanto de la sección uno como la sección dos.

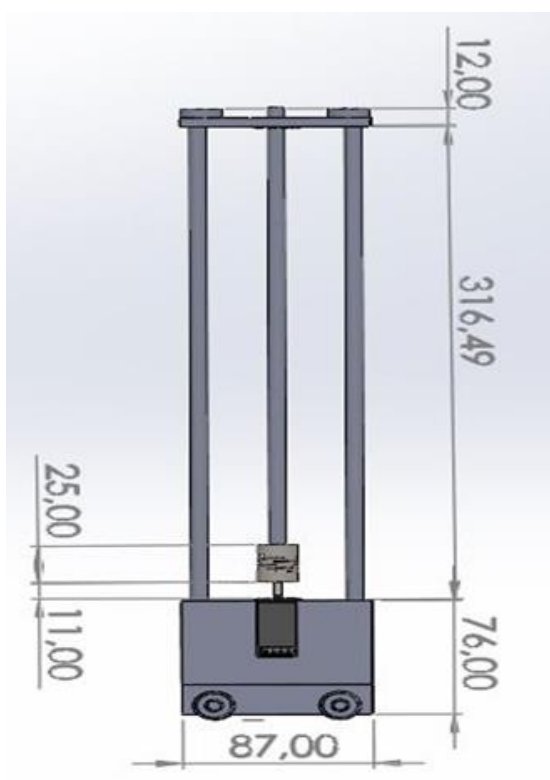

Figura 9. Diseño Sección 2.

Elaboración: Los autores.

Para la rehabilitación se necesitará una estructura que permita moverse conjunto con la mano de manera libre por tal razón se diseñó una placa que será ajustada posteriormente y tienen a su vez bastante amplitud de movimiento. 
Revista Arbitrada Interdisciplinaria KOINONIA

Año VI. Vol VI. N${ }^{\circ}$. Edición Especial: Administración. 2021

Hecho el depósito de Ley: FA2016000010

ISSN: $2542-3088$

FUNDACIÓN KOINONIA (F.K). Santa Ana de Coro. Venezuela.

Elvis Francisco Sinchiguano-Sánchez; Andrea Estefania Cuñez-Yánez; Eduardo Francisco García-Cabezas;

Juan Carlos Cayán-Martínez

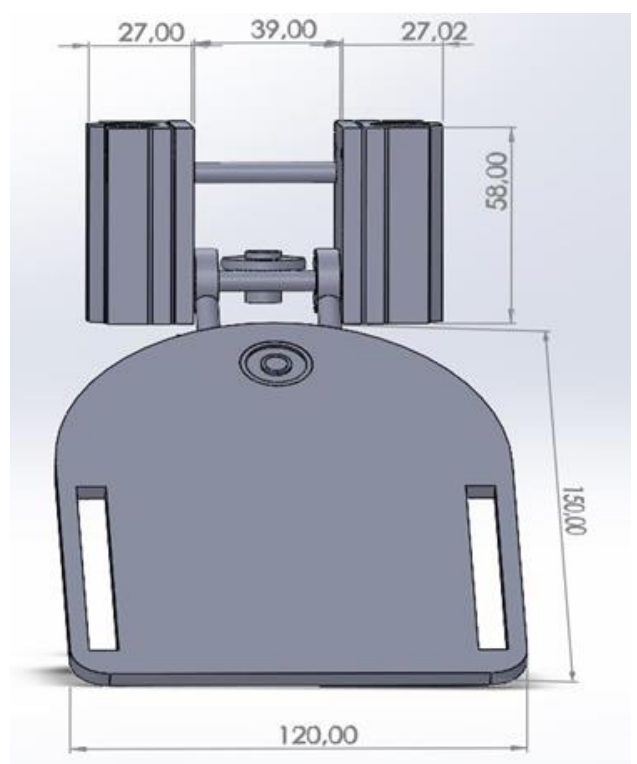

Figura 10. Diseño para la sujeción de la muñeca.

Elaboración: Los autores.

Estructura general estación A movimiento vertical y horizontal, para la estructura se realizó un marco metálico donde se pueda ubicar de manera correcta los motores paso a paso, ejes de transmisión, tornillo sin fin, al igual que las piezas impresas. 
Revista Arbitrada Interdisciplinaria KOINONIA

Año VI. Vol VI. N 1 . Edición Especial: Administración. 2021

Hecho el depósito de Ley: FA2016000010

ISSN: 2542-3088

FUNDACIÓN KOINONIA (F.K). Santa Ana de Coro. Venezuela.

Elvis Francisco Sinchiguano-Sánchez; Andrea Estefania Cuñez-Yánez; Eduardo Francisco García-Cabezas;

Juan Carlos Cayán-Martínez

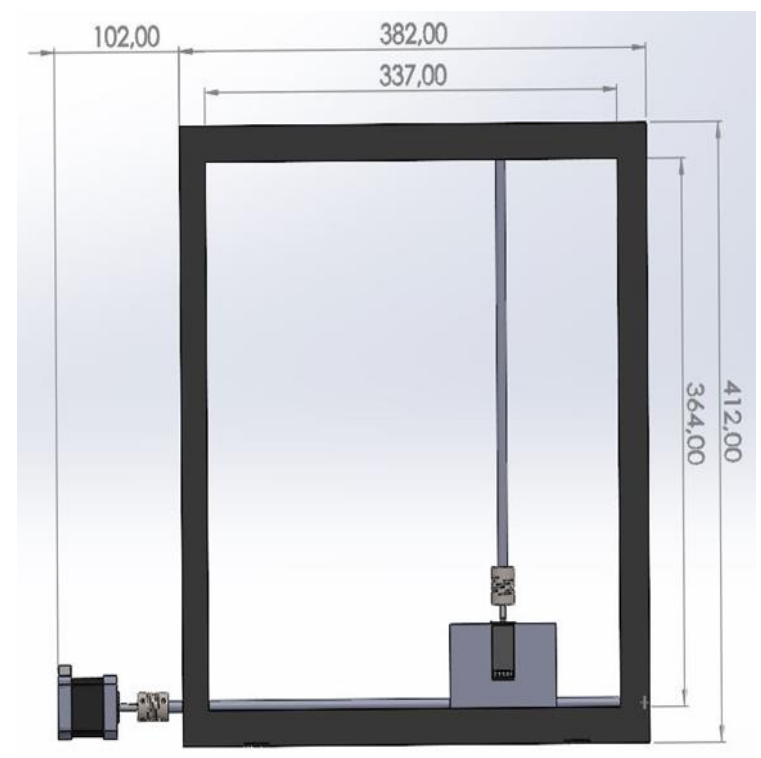

Figura 11. Estructura para el desplazamiento vertical y horizontal. Elaboración: Los autores.

Estación B, en la presente estación se realizó para lo que es la rehabilitación de los dedos de la mano donde se busca el semi agarre y agarre completo también conocido como puño, con los datos adquiridos anteriormente se tiene un dimensionamiento que nos permite tener un rango de medidas para el diseño. El diseño se lo crea con ángulos y medidas de igual forma para generar los movimientos se tiene el mismo principio de la estación A. Un factor a considerar es que los dedos no tienen las mismas medidas por ende en cada dedo se tomó diferente longitud para lo que es el tornillo. 
Revista Arbitrada Interdisciplinaria KOINONIA

Año VI. Vol VI. N¹. Edición Especial: Administración. 2021

Hecho el depósito de Ley: FA2016000010

ISSN: 2542-3088

FUNDACIÓN KOINONIA (F.K). Santa Ana de Coro. Venezuela.

Elvis Francisco Sinchiguano-Sánchez; Andrea Estefania Cuñez-Yánez; Eduardo Francisco García-Cabezas; Juan Carlos Cayán-Martínez

Tabla 5.

Diseño de estructura estación B.

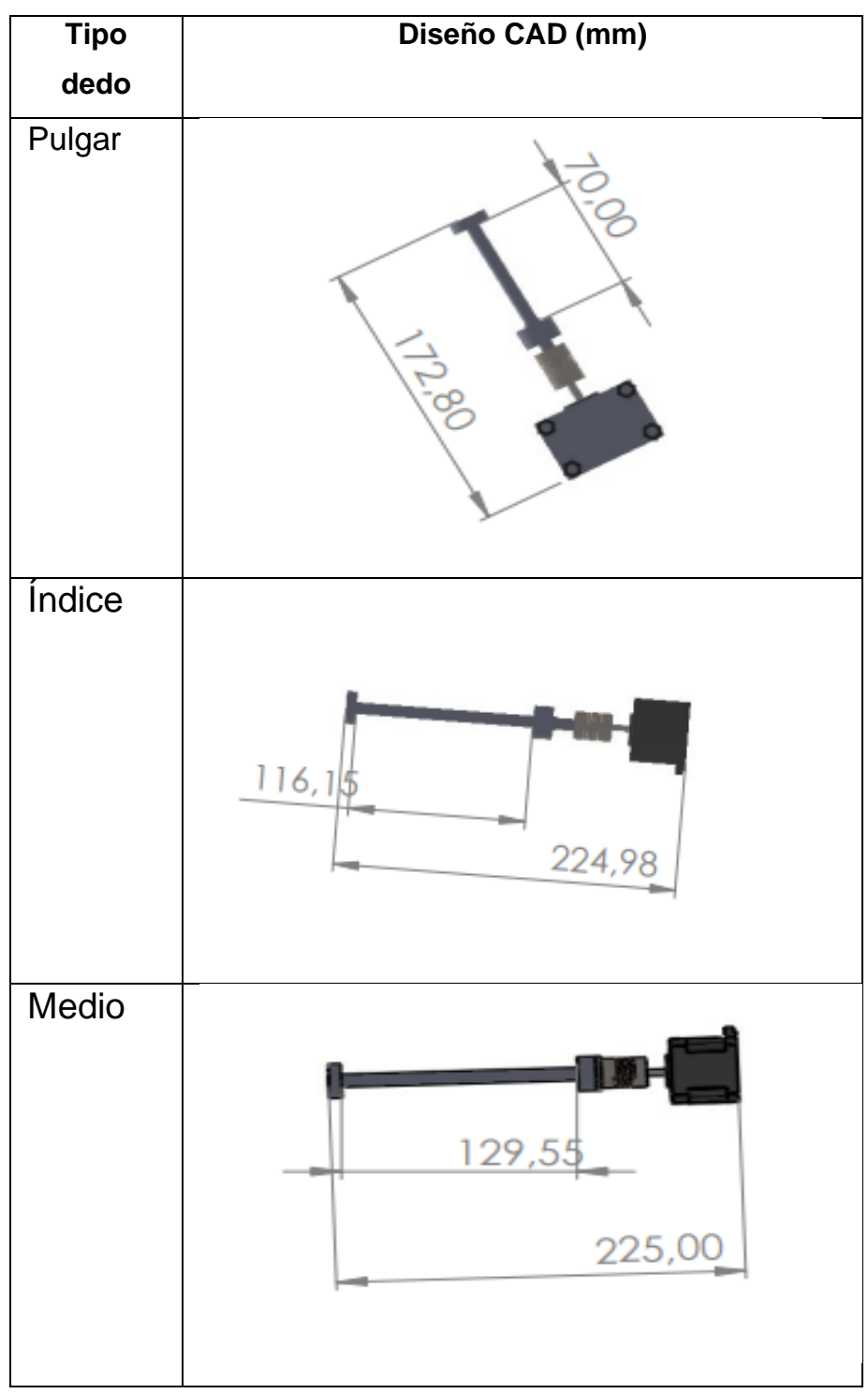


Revista Arbitrada Interdisciplinaria KOINONIA

Año VI. Vol VI. N¹. Edición Especial: Administración. 2021

Hecho el depósito de Ley: FA2016000010

ISSN: 2542-3088

FUNDACIÓN KOINONIA (F.K). Santa Ana de Coro. Venezuela.

Elvis Francisco Sinchiguano-Sánchez; Andrea Estefania Cuñez-Yánez; Eduardo Francisco García-Cabezas;

Juan Carlos Cayán-Martínez

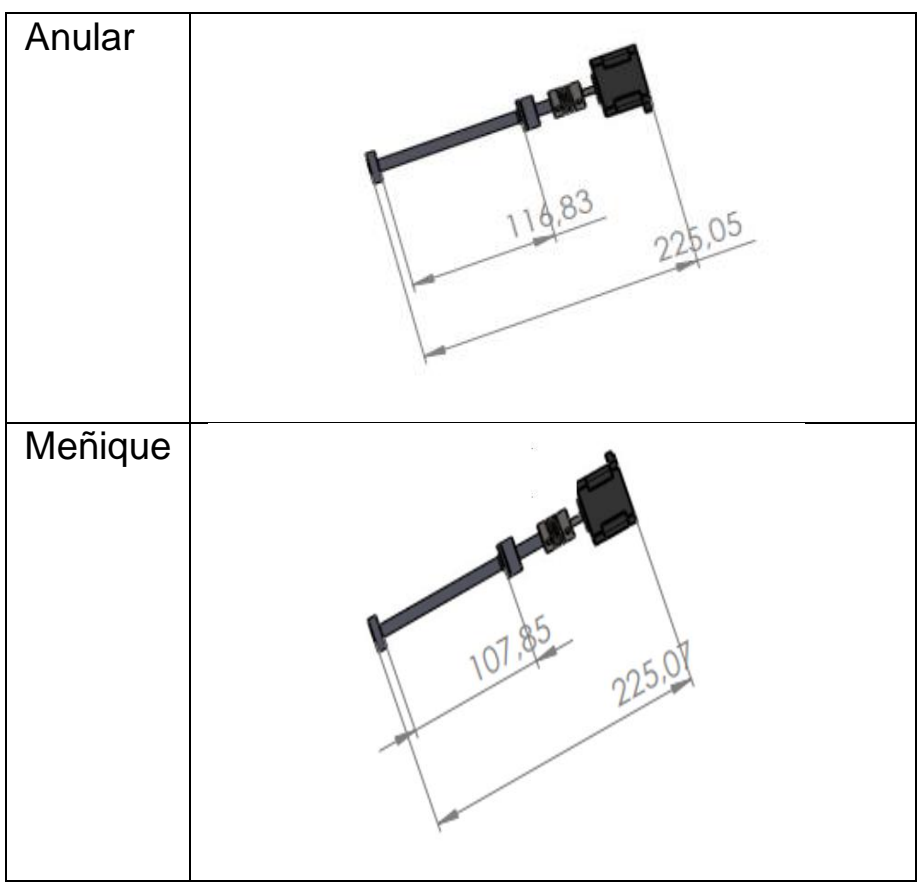

Elaboración: Los autores.

Estructura general estación B, mediante los datos obtenido en la Tabla 1-3, el diseño y ubicación de los tornillos sin fin y sus respectivos motores se puede diseñar lo que es la estructura exterior para la estación.

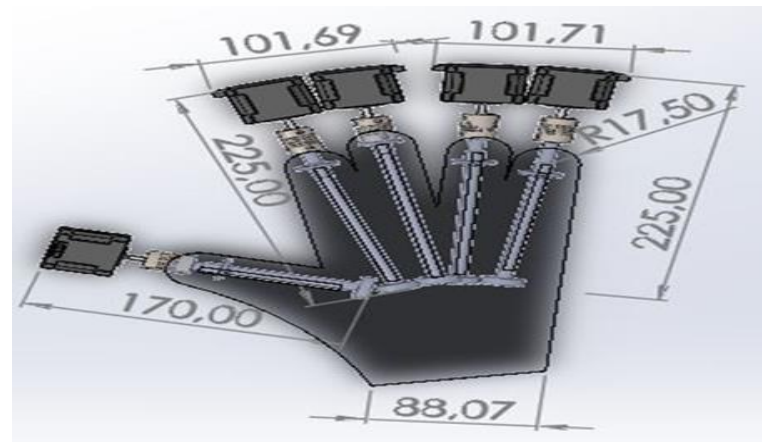

Figura 12. Diseño de la estación $B$.

Elaboración: Los autores. 


$$
\begin{gathered}
\text { Revista Arbitrada Interdisciplinaria KOINONIA } \\
\text { Año VI. Vol VI. Nº1. Edición Especial: Administración. } 2021 \\
\text { Hecho el depósito de Ley: FA2016000010 } \\
\text { ISSN: 2542-3088 } \\
\text { FUNDACIÓN KOINONIA (F.K). Santa Ana de Coro. Venezuela. }
\end{gathered}
$$

Elvis Francisco Sinchiguano-Sánchez; Andrea Estefania Cuñez-Yánez; Eduardo Francisco García-Cabezas;

Juan Carlos Cayán-Martínez

\section{CONCLUSIÓN}

Al finalizar el estudio se logró establecer un protocolo de rehabilitación post intervención de STC, en la que se estableció diferentes movimientos como son: flexión-extensión, desviación radial-cubital y puño de manos, para la adquisición de la base de datos de las medidas de manos se la obtuvo de 23 alumnos de un rango de edad de 19 - 24 años. Asimismo, se, desarrollo el bosquejo teórico para el sistema electromecánico para la inducción de movimientos en la mano y dedos que permitan la ejecución de ejercicios, que cumplen con los movimientos considerados en el protocolo de rehabilitación establecidos para el caso.

Por otro lado, mediante el análisis documental se determinó los medios necesarios para realizar la construcción del sistema de rehabilitación, los cuales son: Hardware y Software., los cuales cumplen con los requerimientos para la funcionalidad del dispositivo desarrollado. Para el desarrollo de un entorno gráfico que permita la interacción del sistema electromecánico y usuario se seleccionó varios softwares como son: LabView, SolidWorks y Arduino, la comunicación entre ellos permite al usuario manejar y configurar el dispositivo electrónico de acuerdo con los requerimientos establecidos para la rehabilitación.

Por lo tanto, para el protocolo de rehabilitación es sustancial realizar un estudio bibliográfico para tener más información ya sea en el ámbito tecnológico, médico, terapéutico, con esto se permitirá tener una selección de ejercicios óptimos y para finalizar la elaboración del sistema se debe considerar diferentes sistemas de transmisión, siempre tomando en cuenta que cumpla con las características apropiadas para la rehabilitación de las personas. 


\section{FINANCIAMIENTO}

No monetario.

\section{AGRADECIMIENTO}

A la Escuela Superior Politécnica de Chimborazo Facultad de Mecánica, por todo el apoyo brindado en la motivación y desarrollo de esta investigación.

\section{REFERENCIAS CONSULTADAS}

Amaya Fariño, L., Tumbaco Reyes, A., Roca Quirumbay, E., Villón González, T., Mendoza Morán, B., y Reyes Quimís, Ángela. (2020). El loT aplicado a la Domótica. [The loT applied to Home Automation]. Revista Científica $Y$ Tecnológica UPSE, 7(1), 21-28. https://doi.org/10.26423/rctu.v7i1.490

Ayala Pozo, S. (2018). Prevalencia de síndrome de túnel carpiano en puestos administrativos. [Prevalence of carpal tunnel syndrome in administrative positions]. Universidad Internacional Sek. Ecuador. Recuperado de: https://n9.cl/c27en

Cabrera González, J., y Roche Gonzélez, E. (2021). Caracterización clínica y electroneuromiográfica de los pacientes con síndrome del túnel carpiano. [Clinical and electroneuromyographic characterization of patients with carpal tunnel syndrome]. Medimay, 28(3), 366-379. Recuperado de: https://n9.cl/8apf2v

Cooper, R., Scott J., y Kleinschmidt, E.(2002) Optimizing the Stage-Gate Process: What Best-Practice Companies Do-I, Research-Technology Management, 45:5, 2127, DOI: $\underline{10.1080 / 08956308.2002 .11671518}$

Fernández Padrón, E., Fernández García, S., y Pérez Barrios, R. (2012). LabVIEW aplicado al diagnóstico de estado en tiempo real de transformadores de potencia. [LabVIEW applied to real-time status diagnosis of power transformers]. Ingeniería Energética, 33(1), 46-58. Recuperado de: https://n9.cl/xw8ph 


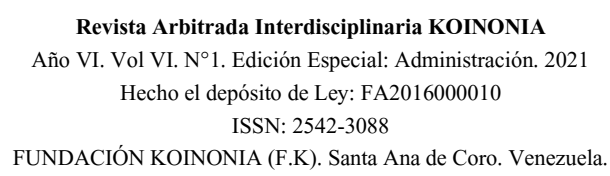

Elvis Francisco Sinchiguano-Sánchez; Andrea Estefania Cuñez-Yánez; Eduardo Francisco García-Cabezas;

Juan Carlos Cayán-Martínez

Jiménez del Barrio, S., Bueno Gracia, E., Hidalgo García, C., Estébanez de Miguel,E.,

Tricás Moreno, J., Rodríguez Marco, S., y Ceballos Laita, L. (2016) Tratamiento conservador en pacientes con síndrome del túnel carpiano con intensidad leve o moderada.[ Conservative treatment in patients with carpal tunnel syndrome with mild or moderate intensity]. Neurología,33(9):590-601.DOI: https://doi.org/10.1016/i.nrl.2016.05.018

Mancisidor, A., Zubizarreta, A., Cabanes, I., Bengoa, P., y Hyung Jung, J. (2018). Dispositivo Robótico Multifuncional para la Rehabilitación de las Extremidades Superiores. [Multifunctional Robotic Device for Upper Extremity Rehabilitation]. Revista Iberoamericana de Automática e Informática industrial, 15(2), 180-191. doi: https://doi.org/10.4995/riai.2017.8820

Peña Ayala, L, Gómez Bull, K., Vargas Salgado, M., Ibarra Mejía, G., y Máynez Guaderrama, A. (2018). Determinación de rangos de movimiento del miembro superior en una muestra de estudiantes universitarios mexicanos. [ Determination of ranges of motion of the upper limb in a sample of Mexican university students]. Revista Ciencias De La Salud, 16, 64-74. https://doi.org/10.12804/revistas.urosario.edu.co/revsalud/a.6845

Rodríguez Menéndez, E., Gonzales Vargas, M., Ticona Anahua, R., Campos Condori, H., Ucharico Chura, E., Ramios Ccallo, J., Calahuille Aycachi, Y., Díaz Mamani, A., Avalos Cueva, A., Velasquez Nina, V., y Cotrado Estalla, E. (2020). Síndrome del túnel carpiano. [Carpal tunnel síndrome]. Revista Médica Basadrina, 14(2), 69-79. https://doi.org/10.33326/26176068.2020.2.1017

Velázquez Sánchez, A., Merchán Cruz, E., Hernández Gómez, L., y Urriolagoitia Calderón, G. (2007). Rango de movilidad y función descriptiva del dedo índice. [ Range of mobility and descriptive function of the index finger]. Científica, 11(4),177-188. Recuperado de: https://n9.cl/kc258

(C2021 por los autores. Este artículo es de acceso abierto y distribuido según los términos y condiciones de la licencia Creative Commons Atribución-NoComercial-Compartirlgual 4.0 Internacional (CC BY-NC-SA 4.0) (https://creativecommons.org/licenses/by-nc-sa/4.0/). 